\title{
Lower energy levels adequate for effective transcleral diode laser cyclo- photocoagulation in Asian eyes with refractory glaucoma
}

Abstract

Purpose To study the treatment parameters for diode laser cyclophotocoagulation (DLCP) in Asian Indian eyes using laser energy titrated to clinical response.

Methods This prospective interventional longitudinal study included 66 eyes of 66 patients with varied aetiology refractory glaucoma, no previous cycloablation, and minimum 1 year follow-up. DLCP was performed using the Oculight ${ }^{\mathbb{R}}$ Diode laser

Department of Ophthalmology, Postgraduate Institute of Medical Education and Research,

Chandigarh, India

Correspondence: SS Pandav,

Department of

Ophthalmology,

Postgraduate Institute of Medical Education and Research,

Sector 12,

Chandigarh 160012,

India

Tel: 91172 2747837;

Fax: 911722747837

E-mail: sspandav@

yahoo.com

Received: 25 July 2006 Accepted in revised form: 2 October 2006

Published online:

1 December 2006

No author has any proprietary interest in any of the products mentioned in this paper system (IRIS ${ }^{\odot}$ Medical Instruments Inc., CA, USA). Power used per spot was titrated according to the audible 'pops' indicating tissue microexplosion. The mean laser energy delivered, post-laser intraocular pressure (IOP) reduction, complications, and requirement of re-treatment in various subgroups were analyzed. Differences in energy delivered in each subgroup were assessed by analysis of variance with post hoc Bonferroni corrections. Linear regression analysis was used to identify possible predictive factors for failure of cyclodiode therapy.

Results The mean total energy delivered per eye was $87.80 \pm 31.8 \mathrm{~J}$ (range $105.4 \pm 36.8 \mathrm{~J}$ in neovascular glaucoma (NVG) to $61.5 \pm 8.8 \mathrm{~J}$ in uveitic glaucoma $(P=0.134))$. Mean pre treatment IOP was $36.4 \pm 10.7 \mathrm{mmHg}$, which reduced to $19.4 \pm 9.8 \mathrm{mmHg}(P<0.001)$ at 1 week, and $15.6 \pm 6.6 \mathrm{mmHg}$ at 1 year. At 1 year, 58 of 66 patients had IOP $<22.0 \mathrm{mmHg}$ (response rate $87.8 \%$ ), and six patients had hypotony (success rate $\mathbf{7 8 . 8 \%}$ ). The uveitic glaucoma group had $100 \%$ success rate. NVG group required maximum re-treatments. Conclusions DLCP with a titrated energy protocol needs resulted in lower energy in
Asian Indian eyes compared to that reported in literature, and different energy levels are needed for different diseases. 'Standard treatment parameters' for DLCP may be inappropriate for all diseases and all races. Eye (2008) 22, 398-405; doi:10.1038/sj.eye.6702653; published online 1 December 2006

Keywords: diode laser; cyclophotocoagulation; transcleral; refractory glaucoma

\section{Introduction}

Cyclodestructive procedures for intractable glaucoma unlikely to benefit from surgery, have evolved in the last 70 years: from penetrating cyclodiathermy, ${ }^{1}$ to cyclocryotherapy, ${ }^{2-4}$ to ultrasound for ciliary body ablation, ${ }^{5,6}$ to laser cyclophotocoagulation. ${ }^{7-24}$ The chief drawback of these procedures as a group is a narrow therapeutic window, which implies that there is a very small safety zone in which it is effective without causing significant complications.

Currently, transcleral laser cyclophotocoagulation (TSCPC) procedures appear to provide the best combination of effectiveness, portability, expense, and ease of use.

Since Beckman et $\mathrm{al}^{\prime} \mathrm{s}^{7}$ first report of transcleral cyclophotocoagulation using Ruby laser $(693 \mathrm{~nm})$, a wide variety of laser wavelengths has been used. The energy uptake appears to be influenced by melanin absorption. ${ }^{10}$ The most frequently used lasers for this modality of treatment are the $1064 \mathrm{~nm}$ $\mathrm{Nd}$ :Yag lasers ${ }^{8-11}$ and $810 \mathrm{~nm}$ semiconductor diode. ${ }^{12-24}$ Both wavelengths can produce 
thermal tissue damage, and there is evidence that the semiconductor diode laser with $810 \mathrm{~nm}$ wavelength exhibits less scleral transmission and considerably greater absorption by melanin compared to the $1064 \mathrm{~nm}$ Nd:Yag laser. ${ }^{25}$ The clinically significant effect is that the energy needed to produce comparable lesions is less with the diode laser compared to that required by the Nd:YAG laser. ${ }^{12}$ Owing to the same reason, less laser energy has also been reported to be needed in pigmented eyes, to produce the same effect. ${ }^{10,11}$

Although there are numerous reports describing the effectiveness of TSCPC, an accurate comparison of different techniques based on published studies is difficult because of wide variation in treatment parameters and lack of uniform definition of success. There are no standard treatment parameters defined, and the laser energy delivered varies considerably.

We conducted this study to assess treatment parameters of diode laser cyclophotocoagulation (DLCP) in our population of Asian Indian eyes and to see whether the energy delivered in these pigmented eyes differed from that reported in literature.

\section{Material and Methods}

This prospective longitudinal study was undertaken in patients with refractory glaucoma who had not responded to or were unlikely to benefit from glaucoma surgery. Patients were recruited from the Glaucoma Clinic of the Postgraduate Institute of Medical Education and Research, Chandigarh, India. Ethical clearance was obtained from the Institute's Ethics Review Committee and the study adhered to the principles enshrined in the Declaration of Helsinki Principles.

The study group comprised patients who had never received any form of cycloablative treatment, had a bestcorrected visual acuity (BCVA) $<3 / 60$, and fulfilled one or more of the following inclusion criteria:

(a) medically uncontrolled glaucoma patients who were unlikely to respond to or declined filtering surgery;

(b) poor visual potential in eyes with poor prognosis for filtration surgery (eg prior pars-plana vitrectomy $(\mathrm{PPV})$, penetrating keratoplasty (PK) with failed graft, repeated failed glaucoma surgery, chemical burns, etc.);

(c) painful blind eyes with high intraocular pressure (IOP).

Patients fulfilling the eligibility criteria presenting to our Clinic between July 2004 and January 2006 were consecutively enrolled for the study. Baseline information included patients' age, gender, race, type of glaucoma, underlying ocular disease, BCVA, IOP, number of topical and oral anti-glaucoma medications, and previous glaucoma and/or other ocular surgery.

\section{DLCP procedure}

After taking informed consent, the TSCPC procedure was performed under a peribulbar block with anaesthetic mixture of $2 \%$ lidocaine hydrochloride and $0.5 \%$.

bupivacaine. A lid speculum was used to separate the eyelids. The laser energy was delivered through a $600 \mu \mathrm{m}$ diametre quartz fibre oriented within the G-probe handpiece of the Oculight ${ }^{\circledR}$ Diode laser system (IRIS Medical Instruments Inc., Mountainview, CA, USA) to centre the treatment $1.2 \mathrm{~mm}$ behind the limbus. The fibre optic tip protrudes $0.7 \mathrm{~mm}$ from the G-probe contact surface in order to indent the conjunctiva and sclera, thereby improves the laser transmission to the ciliary body. Care was taken to apply the G-probe to the limbus indenting as above as this ensured that the G-probe surface contour matched the scleral curvature, and the posterior angulation was correctly oriented to protect the lens of phakic eyes from laser damage. Three quarters of the circumference of the ciliary body (six per quadrant for 270 degrees resulting in 18 spots) was treated, and the supero-nasal quadrants were spared in all cases.

The energy delivery was started at $1750 \mathrm{~mW}$ for $2 \mathrm{~s}$ and increased in $250 \mathrm{~mW}$ intervals till an audible 'pop' was heard (which indicates tissue disruption ${ }^{25,26}$ ), following which the power was reduced by $250 \mathrm{~mW}$ until the 'pops' were no longer audible, and the treatment was completed at these parameters. The total cyclodiode energy (joules) delivered per session was calculated by multiplying the number of laser burns by the duration (seconds) and power (Watts).

If the patient was on oral acetazolamide before laser treatment, it was continued for a period of 1 week after laser treatment. At 1 week post-laser treatment, oral acetazolamide was discontinued if the IOP was $<22 \mathrm{mmHg}$, were continued topical IOP lowering medications depending on the response. Topical steroids, usually dexamethasone $0.1 \%$, four times a day, and cycloplegics (atropine 1\%) three times a day, were prescribed for 2-4 weeks after treatment.

If an adequate response was not achieved in 6 weeks, cyclodiode was repeated up to a maximum of two treatment sessions. The treated quadrants were re-treated each time, leaving the supero-nasal quadrant totally free of cycloablation. The total energy delivered to an eye was calculated by adding the energy delivered in all treatment sessions.

Follow-up examinations were performed weekly for first month, monthly for 3 months, three monthly for the first year, and six monthly thereafter. At every follow-up examination, visual acuity, IOP, anti-glaucoma 
medication used, slit-lamp biomicroscopic appearance, and complications were recorded. Only patients completing 1-year follow-up were included in the final analysis.

The outcome of cyclodiode therapy was determined in terms of

1. Success rate: defined as the percentage of eyes achieving an IOP between 5 and $21 \mathrm{mmHg}$ with or without topical medication in all eyes following cyclodiode therapy at their final follow-up visit.

2. Response rate: defined as the percentage of patients achieving an IOP of $<22 \mathrm{mmHg}$ or $>30 \%$ drop in IOP and included eyes that developed hypotony (IOP $<5 \mathrm{mmHg}$ ).

3. Cyclodiode efficacy index; $;^{19}$ defined as the ratio of the response rate (expressed as a decimal) to the mean number of treatment sessions. Thus, a cyclodiode efficacy index of 1.0 would indicate that all the eyes in an evaluated group achieved a satisfactory IOP response following a single treatment episode.

Data were recorded on prospectively filled patient forms.

\section{Statistical methods}

Sample size calculation

The aim of this study was to compare the success in our patients with that reported in literature. Reported success rates vary from 66 to $80 \%$ in Caucasians ${ }^{15,18,19}$ to $92 \%$ in Indian eyes. ${ }^{20}$ Taking a mean reported success rate of $70 \%$ and a difference of at least $20 \%$ to be significant, a study with $80 \%$ power and $5 \%$ significance would require a minimum of 63 patients, by the following formula:

$$
\text { Minimum sample size }=\frac{2 \times(1.96+0.84)^{2} \times 80 \times 20}{20^{2}}
$$$$
=62.72
$$

\section{Statistical analysis}

Results were analyzed using the SPSS for Windows software, Version 10.0, ${ }^{\circ}$ SPSS Inc., Chicago, US. Differences in pre treatment and final IOP and pre- and post-treatment topical anti-glaucoma drug use were analysed using the Wilcoxon Signed Rank Test. The difference in pre- and post-treatment systemic carbonic anhydrase inhibitor use was compared using the McNemar test. The differences in energy delivered to eyes in each subgroup were assessed by analysis of variance with post hoc Bonferroni corrections. Linear regression analysis was used to identify possible predictive factors for failure of cyclodiode therapy such as age, gender, pretreatment IOP, and diagnosis. The results were considered significant at $P<0.05$.

\section{Results}

In total, 71 eyes of 71 eligible patients, were recruited for the study. Five patients were lost to follow-up after the first 3 months and were excluded from the study group. Data from 66 eyes of 66 patients were analysed. Mean follow-up was $14.3 \pm 2.2$ months. Baseline characteristics are detailed in Table 1. The mean age was $47.2 \pm 17.3$ years and 52 patients were male. Neovascular glaucoma (NVG) accounted for the largest subgroup (27.3\%) of patients.

The mean total energy delivered per eye was $87.80 \pm 31.8 \mathrm{~J}$. The energy delivered in each subgroup is detailed in Table 2. The mean total energy delivered ranged from $105.4 \pm 36.8 \mathrm{~J}$ in the NVG group to $61.5 \pm 8.8 \mathrm{~J}$ in the uveitic glaucoma group, but these differences did not reach statistical significance $(P=0.134)$. Eleven patients required one re-treatment, which meant that 66 eyes required 77 treatments, translating into mean 1.16 sessions per eye. There was no significant difference in the energy delivered between the first and second treatments. Of the eyes needing re-treatment, five eyes had NVG, two eyes had post-PK glaucoma, one each had primary angle closure glaucoma (PACG), primary open angle glaucoma (POAG), post-PPV glaucoma, postscleral buckling (SB) glaucoma, and traumatic glaucoma.

The mean pre treatment IOP was $36.4 \pm 10.7 \mathrm{mmHg}$, which reduced significantly $(P<0.001)$ to

Table 1 Baseline characteristics of patient

\begin{tabular}{lc}
\hline Gender & No $(\%)$ \\
\hline Males & $52(78.8)$ \\
Females & $14(21.2)$ \\
Age (years) & \\
$\quad$ (Mean \pm SD) & $47.2 \pm 17.3$ \\
(Range) & $(13-72)$ \\
Diagnosis & \\
Neovascular glaucoma & \\
Post-penetrating keratoplasty & $18(27.3)$ \\
Post-PPV glaucoma & $12(18.2)$ \\
Uveitic glaucoma & $8(12.1)$ \\
Traumatic glaucoma & $6(9.1)$ \\
POAG & $6(9.1)$ \\
Post-scleral buckle & $6(9.1)$ \\
Angle closure glaucoma & $5(7.6)$ \\
Buphthalmos & $3(4.5)$ \\
& $2(3.0)$ \\
Total & $66(100)$ \\
\hline
\end{tabular}


$19.4 \pm 9.8 \mathrm{mmHg}$ at the first week following treatment, and was maintained at $15.6 \pm 6.6 \mathrm{mmHg}$ at 1 year followup (Figure 1). Details of IOP reduction in all subgroups are depicted in Table 3 . There was no significant difference in the pre-treatment IOP $(P=0.166)$ or the final IOP at 1 year $(P=0.653)$ between any of the subgroups.

Before treatment, 49 patients $(74.2 \%)$ required oral acetazolamide for IOP control, and the number of patients with IOP decreased to 37 (56.1\%), 31 (47\%), and $16(24.2 \%)$ patients at 1 week, 1 month, and 3 months, respectively. Of these 49 patients, NVG comprised the largest subgroup (17 patients) followed by postpenetrating keratoplasty (PK), post-pars-plana vitrectomy (PPV), and post-traumatic glaucoma (6 patients each). No patient required acetazolamide after from the 6 months follow-up $(P<0.000$; Figure 1$)$. Dependence on topical anti-glaucoma medication also reduced significantly. Mean topical anti-glaucoma drug requirement decreased from $2.6 \pm 0.9$ before treatment to $2.1 \pm 1.1$ at 3 months and to $1.8 \pm 1.2$ (Figure 1 ) at the end of 1 year - the differences were statistically significant ( $P=0.020$ and $P<0.001$, respectively).

At 1 year follow-up, 58 of 66 patients had IOP less than $22.0 \mathrm{mmHg}$ with or without topical drugs, translating a response rate of $87.8 \%$ (Table 2 ). Six patients had final IOP $<5.0 \mathrm{mmHg}$. Thus the outcome in terms of success was $78.8 \%$ (52/66). Outcome in all patient subgroups is detailed in Table 2. Uveitic glaucoma, post-PPV glaucoma, and congenital glaucoma showed the best response rate $(100 \%)$, but one patient each in the PPV and congenital glaucoma group developed hypotony. The Uveitic glaucoma group had 100\% success rate, followed by post-PPV group with $87.5 \%$. Post-traumatic glaucoma $(66.7 \%)$ and PACG $(66.7 \%)$ had the least success rates. However, these differences did not reach statistical significance $(P=0.81)$.

Table 2 Energy delivered and outcome of DLCP in all subgroups

\begin{tabular}{lcccccc}
\hline Diagnosis & $\begin{array}{c}\text { Total energy } \\
\text { delivered per } \\
\text { eye }(J)\end{array}$ & $\begin{array}{c}\text { Re-treatment } \\
\text { sessions (no) }\end{array}$ & $\begin{array}{c}\text { Mean treatments } \\
\text { per patient (no) }\end{array}$ & $\begin{array}{c}\text { Response } \\
(\text { IOP<22.0 mmHg) } \\
n o(\%)\end{array}$ & $\begin{array}{c}\text { Success (IOP } \\
5-22 \text { mmHg) } \\
n o(\%)\end{array}$ & $\begin{array}{c}\text { Cyclodiode } \\
\text { efficacy index }\end{array}$ \\
\hline All patients $(n=66)$ & $87.8 \pm 31.8$ & 11 & 1.16 & $58(87.8)$ & $52(78.8)$ & 0.758 \\
NVG $(n=18)$ & $105.4 \pm 36.8$ & 5 & 1.3 & $15(83.3)$ & $13(72.2)$ & 0.64 \\
Post-PK $(n=12)$ & $83.2 \pm 31.2$ & 2 & 1.2 & $11(91.7)$ & $10(83.3)$ & 0.77 \\
Post-PPV $(n=8)$ & $85.5 \pm 24.5$ & 1 & 1.1 & $8(100.0)$ & $7(87.5)$ & 0.91 \\
Uveitis $(n=6)$ & $61.5 \pm 8.8$ & 0 & 1.0 & $6(100.0)$ & $6(100.0)$ & 1.0 \\
Trauma $(n=6)$ & $85.5 \pm 34.5$ & 1 & 1.2 & $5(83.3)$ & $4(66.7)$ & 0.69 \\
POAG $(n=6)$ & $84.0 \pm 32.0$ & 1 & 1.2 & $5(83.3)$ & $5(83.3)$ & 0.69 \\
SB $(n=5)$ & $93.6 \pm 33.4$ & 1 & 1.2 & $4(80.0)$ & $4(80.0)$ & 0.67 \\
PACG $(n=3)$ & $72.0 \pm 9.0$ & 0 & 1.0 & $2(66.7)$ & $2(66.7)$ & $1(50)$ \\
Congenital & $76.5 \pm 6.4$ & 0 & 1.0 & & & 0.67 \\
glaucoma $(n=2)$ & & & & & & 1.0
\end{tabular}

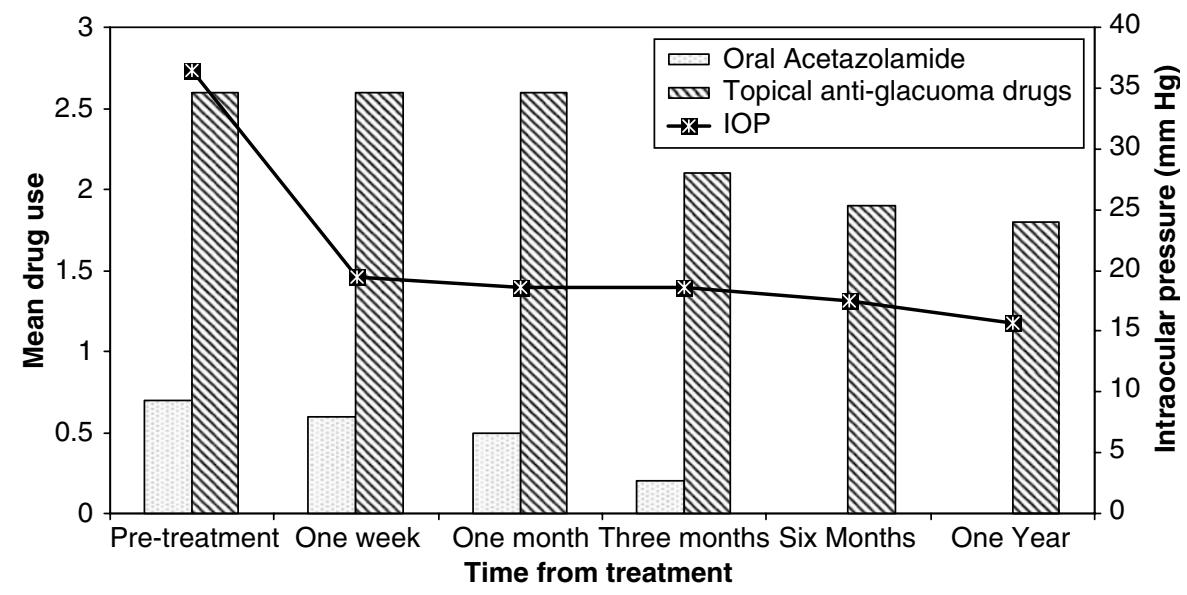

Figure 1 Oral and topical anti-glaucoma drug use with IOP reduction over time. 
Table 3 IOP change in various subgroups over time

\begin{tabular}{|c|c|c|c|c|c|}
\hline Diagnosis & $\begin{array}{c}\text { Pretreatment } \\
\text { Mean IOP }(\mathrm{mmHg})\end{array}$ & $\begin{array}{c}\text { One week } \\
\text { Mean IOP }(\mathrm{mmHg})\end{array}$ & $\begin{array}{c}\text { One month } \\
\text { Mean IOP }(\mathrm{mmHg})\end{array}$ & $\begin{array}{c}\text { Six months } \\
\text { Mean IOP }(m m H g)\end{array}$ & $\begin{array}{c}\text { One year } \\
\text { Mean IOP }(\mathrm{mmHg})\end{array}$ \\
\hline All patients $(n=66)$ & 36.4 & 19.4 & 18.6 & 17.5 & 15.6 \\
\hline NVG $(n=18)$ & 42.9 & 21.3 & 21.1 & 17.1 & 15.5 \\
\hline Post-PK $(n=12)$ & 33.7 & 22.5 & 22.1 & 18.4 & 15.6 \\
\hline Post-PPV $(n=8)$ & 31.0 & 16.4 & 11.4 & 14.8 & 13.3 \\
\hline Uveitis $(n=6)$ & 34.7 & 15.3 & 12.0 & 13.0 & 13.3 \\
\hline Trauma $(n=6)$ & 38.8 & 19.3 & 20.0 & 23.0 & 18.3 \\
\hline POAG $(n=6)$ & 33.3 & 13.7 & 15.3 & 16.0 & 15.3 \\
\hline $\mathrm{SB}(n=5)$ & 35.2 & 24.6 & 21.4 & 21.6 & 18.6 \\
\hline ACG $(n=3)$ & 31.3 & 13.3 & 19.3 & 22.6 & 19.3 \\
\hline Congenital glaucoma $(n=2)$ & 32.0 & 21.0 & 11.0 & 10.0 & 10.0 \\
\hline
\end{tabular}

The overall cyclodiode efficacy index was 0.758 (0.88/ 1.16 ), meaning that $75.8 \%$ of patients in our study group achieved satisfactory IOP control following a single treatment session. The cyclodiode efficacy index is depicted group wise in Table 2. The uveitic and congenital glaucoma groups were most sensitive to DLCP with cyclodiode efficacy index of 1.0, whereas the NVG group was the least sensitive with a cyclodiode efficacy index of 0.64 .

Post-laser complications included pain requiring oral analgesics in $30(45.5 \%)$ eyes, hyphema in two (3.03\%) eyes (both eyes had neovascular glaucoma), and hypotony in six $(9.09 \%)$ eyes, not related to the subtype of glaucoma. The post-intervention IOP course in these six patients is depicted in Figure 2. All patients except two showed IOP control by 1 month after intervention, but developed hypotony by 3 months. One patient with NVG and one with post-traumatic glaucoma were re-treated at 6 weeks for persistent raised IOP, and developed hypotony subsequently.

A total 63 patients preserved their pre treatment visual acuity, whereas it deteriorated in three patients from counting fingers to light perception. No eye lost light perception vision after DLCP.

Linear regression analysis model for possible predictors of response to cyclophotocoagulation is shown in Table 4 . The response was significantly related to only pre-treatment IOP $(P=0.030)$, whereas the type of glaucoma, age, or gender had no bearing on the final outcome.

\section{Discussion}

The goal of transcleral cyclophotocoagulation is to significantly reduce the IOP whereas minimizing damage to adjacent tissues and preventing hypotony. As the effectiveness of laser energy appears to be related to melanin absorption, ${ }^{10,11}$ it may translate into lower energy levels required in pigmented eyes. However, a simple comparison with the numerous published DLCP

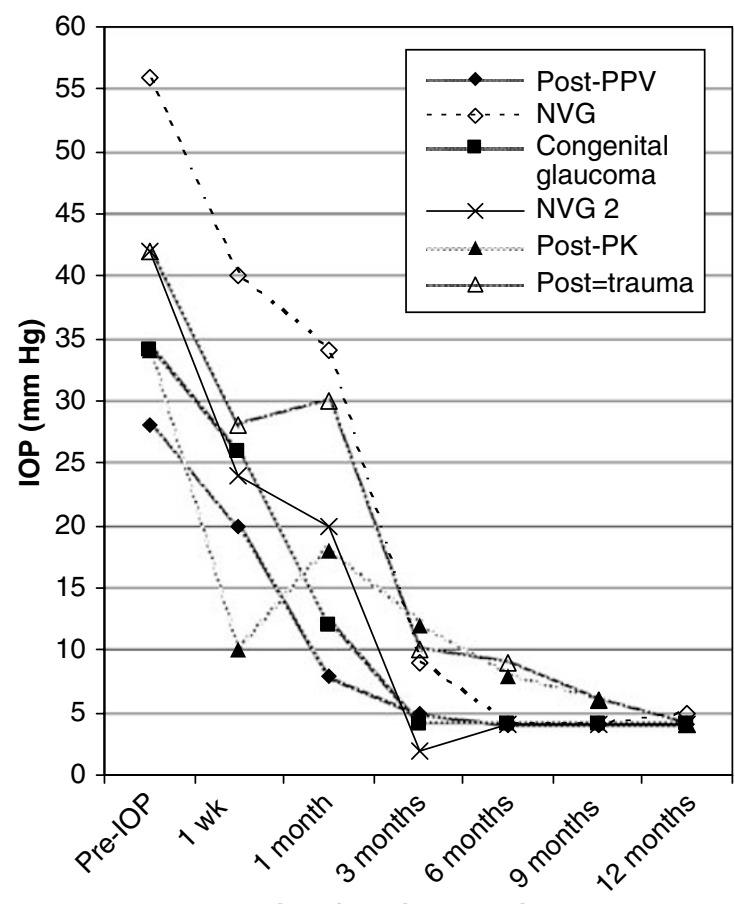

Time from intervention

Figure 2 Course of IOP reduction following DLCP in the six patients who developed hypotony.

Table 4 Linear regression analysis model of predictive factors for response to cyclophotocoagulation

\begin{tabular}{lcc}
\hline Independent factors & $\begin{array}{c}\text { Pearson correlation } \\
\text { coefficient }\end{array}$ & $\begin{array}{c}\text { Significance } \\
(\mathrm{P})^{\mathrm{a}}\end{array}$ \\
\hline Age & 0.104 & 0.630 \\
Gender & 0.034 & 0.714 \\
Diagnosis & 0.060 & 0.248 \\
Baseline IOP & 0.257 & 0.030 \\
\hline
\end{tabular}

${ }^{a}$ Linear regression analysis; dependant variable: response to treatment.

studies is difficult owing to differences in treatment protocols, follow-up, times and success definitions. We, therefore, attempted to compare our results to those 
studies in other populations, that analysed using outcome measures similar to our study.

We did not use uniform treatment parameters for all eyes in our study, but titrated the laser energy delivered by the sound of audible 'pops'. These 'pops' characterize intraocular uveal micro-explosion and represent boiling of tissue water. ${ }^{27}$ Post-operative irido-cyclitis is reportedly more severe with increased 'pops' ${ }^{26}$ Such extensive destruction in the ciliary body region may not be desirable, and may result in increased risk of complications such as hypotony or phthisis. Therefore, our treatment protocol was strictly designed to limit power to a level where these 'pops' were not audible.

In our study, the mean energy delivered per eye was $87.8 \pm 31.8 \mathrm{~J}$, with a response rate of $88 \%$, success rate of 78 , and $16.7 \%$ rate of re-treatment. The mean IOP reduced by $20.8 \mathrm{mmHg}(57.1 \%)$ at the end of 1 year, and all patients were free of oral acetazolamide by 6 months following treatment. These results were more favourable compared to another study in Indian eyes by Gupta and Agarwal. ${ }^{20}$ Of the 52 patients they enrolled, only 22 completed 1 year follow-up. They used 120-160 joules per eye at each session, and treated 360 degrees of the limbus. The success rate was $55.7 \%$ at 2 months, and 22 patients $(42 \%)$ required re-treatment.

Chang et al, ${ }^{21}$ in their study on Chinese eyes, compared two treatment protocols with different energy levels and varied the number of laser applications. One protocol used 135J per session with 27 applications, whereas the other protocol used $165 \mathrm{~J}$ with 55 applications. By 6 months, there was no significant difference in mean IOP reduction between the two groups (19.1 and $14.2 \mathrm{mmHg}$, respectively), but there were far more complications in the group receiving greater energy (hyphema: 23 vs 7; $8 \%$ exudation $v$ s no exudation in the less-energy group). In contrast, our study showed better results with lower energy delivery, and fewer laser applications. As Chinese eyes are also pigmented, many laser applications may have resulted in far greater energy delivery than required for adequate IOP control.

Noureddin et $a^{22}$ reported their results in West Asian eyes, which are also more pigmented than Caucasian eyes. They used a standard 'aggressive' protocol for all eyes delivering $126 \mathrm{~J}$ per session, without titrating for audible 'pops'. The mean IOP fall was $53 \%$ at 1 year. However, vision worsened in $22 \%$ of their patients, and $25 \%$ needed re-treatment. With our 'titrated' energy delivery protocol, 63 of our 66 patients (95.5\%) maintained their pre treatment visual acuity, whereas it worsened in three patients, again highlighting the fact that aggressive protocols may not confer too many advantages, but may lead to unacceptably higher rate of complications.
Murphy et al, ${ }^{19}$ in their study on Caucasian eyes reported results similar to our study, but with higher energy levels. The mean laser energy delivered was $104.1 \pm 37.5 \mathrm{~J}$ per session. The IOP drop was $23.0 \mathrm{mmHg}$ from pre treatment levels $(52.6 \%)$, the mean response rate was $89 \%$, success rate was $79.5 \%$, and the mean cyclodiode efficacy index was 0.59 . More than one-third of their patients $(34.2 \%)$ needed to be re-treated. We had similar outcomes, but the cyclodiode efficacy index was 0.76 , meaning that our patients were more sensitive to DLCP. In both studies, NVG patients required the maximum and uveitis patients required the least energy, but the quantum of energy required in our population was much less. This comparison probably illustrates most clearly that nonpigmented eyes require more energy and more re-treatments for the same effect.

Conversely, there is evidence that suggests that comparable energy levels used in Caucasian and pigmented eyes resulted in better outcomes in the latter. Walland ${ }^{23}$ compared two 'standard' treatment protocols in eyes that were assigned to either group according to the pre treatment IOP and previous cyclodestructive treatment. The 'full' treatment group (which included those with baseline IOP $>30.0 \mathrm{mmHg}$ and no previous cycloablation) received $90 \mathrm{~J}$ per session. Seven of the 22 patients $(32 \%)$ in this group required re-treatment. Success rate (including hypotony) was $59.1 \%$ and response rate ( $>30 \%$ reduction of IOP) was $77 \%$. This study in Australian eyes showed less successful outcomes and greater number of re-treatments than our patients, when similar energy levels were used.

Spencer and Vernon ${ }^{24}$ reported their results in white patients using less energy by virtue of less number of laser applications (14 per eye). The mean energy delivered was $55.5 \pm 1.4 \mathrm{~J}$ per session. Although the mean IOP drop was $15.1 \mathrm{mmHg}$ at 1 year, 26 of their 58 eyes (45\%) required to be re-treated. The lower power used in their study was most likely inadequate, which resulted in more re-treatments for appropriate IOP reduction.

In our study, uveitic eyes were most sensitive to DLCP, whereas eyes with NVG were most resistant. Mean energy levels delivered differed markedly in eyes with uveitic and rubeiotic glaucoma $(61.5 \pm 8.8$ vs $105.4 \pm 36.8 \mathrm{~J}$, respectively), although the difference did not reach statistical significance. Uveitic eyes probably have compromised ciliary body function owing to longstanding cyclitis, as a result of which they respond readily to cycloablation of the remaining functional part of the ciliary body. The 'pops' in eyes with uveitic glaucoma were audible at lower energy levels compared to other subgroups. Probably there is also a degree of ciliary body atrophy in these eyes, which may explain tissue explosion at lower energy levels. All these eyes had a successful outcome with a single treatment session. 
In contrast, of the 11 eyes requiring re-treatment, five had NVG. Although the ultimate success rate in these eyes seemed better than that in eyes with trauma, angle closure glaucoma, and congenital glaucoma, a true comparison would probably not be appropriate owing to the small number of patients in the latter subgroups.

Experimental reports have described ciliary body atrophy with abnormal ciliary epithelium 4 weeks after cyclophotocoagulation. ${ }^{28}$ Most clinical studies ${ }^{15,19}$ have considered 4-6 weeks as the time frame for the response of DLCP, after which the eyes are re-treated. We waited for 6 weeks before re-treating our patients. However, as our study shows, acetazolamide use decreased upto 3 months following treatment, suggesting that one may wait for this much time before declaring the procedure a failure and re-treating the patient.

DLCP is reportedly less successful in younger individuals, ${ }^{15,20}$ and they appear to recover from treatment more rapidly compared to adults. ${ }^{29}$ In our study, there were only two patients with congenital glaucoma, both responded to treatment, and one patient developed hypotony. However, one cannot read much into data from just two patients, and these results should not be commented upon.

The most dreaded complication of ciliary ablation is hypotony and phthisis. In eyes with extremely limited outflow facility, reducing aqueous production to a level that would result in 'normal' IOP on no medication increases this risk manifold. This is one of the reasons for the narrow therapeutic window of cyclodestructive procedures. However, most studies with DLCP have reported low risk of these complications, ${ }^{15-17}$ especially when the treatment protocol involved titrating energy delivery by audible pops. Six of our 66 patients developed hypotony, but there was no alteration in structural integrity of these eyes. Most IOP reductions were seen to occur by 1 month, with hypotony manifesting most commonly by 3 months. One patient each with NVG and post-PK glaucoma were re-treated at 6 weeks for persistently raised IOP. The numbers are too small to comment upon whether this in fact was the cause of the hypotony, but it does suggest that it may be prudent to wait at least 3 months before re-treating.

One important consideration of cyclodestruction is providing freedom from oral acetazolamide, which is a practical concern for many ophthalmologists using this treatment modality. In the present study, $74 \%$ of patients were dependant on oral acetazolamide before treatment, all of whom could be taken off the drug by 6 months. The number of topical drugs required for IOP control also reduced significantly in all subgroups of patients. Other studies have also demonstrated $100 \%$ freedom from oral acetazolamide following DLCP, ${ }_{1}^{15,30}$ whereas in the study by Spencer and Vernon ${ }^{24}$ (who used a lower energy treatment protocol), 91\% of patients were free from oral acetazolamide.

Only the pre -treatment IOP was a significant factor predictive of response to DLCP. Eyes with higher IOP responded better to treatment. Schlote et al ${ }^{15}$ demonstrated a significant association between age and success, whereas in the study by Murphy et $\mathrm{al}^{19}$ male sex was associated with failure of DLCP. We found no such association with age or gender, but the less number of patients in our study needs to be kept in mind before drawing any definitive conclusions.

To summarize, our study demonstrates good results of DLCP with a treatment protocol strictly titrated according to the audible evidence of intraocular tissue explosion. Pigmented eyes appeared to require less energy level than that reported in Caucasian eyes with comparable outcome. DLCP in Asian eyes with lesser energy levels appears to be a safe and effective treatment modality for IOP control in eyes with intractable glaucoma. Larger studies with longer follow-up periods will answer questions pertaining to its long-term efficacy.

\section{References}

1 Vogt A. Versuche zur intraokularen druckherabsetzung mittelst diathermieschädigung des corpus ciliare (Zyklodiathermiestichelung). Klin Monatsbl Augenheilkd 1936; 97: 672-673.

2 Bietti G. Surgical intervention on the ciliary body; new trends for the relief of glaucoma. JAMA 1950; 142: 889-897.

3 Brindley G, Shields MB. Value and limitations of cyclocryotherapy. Graefes Arch Clin Exp Ophthalmol 1986; 224: 545-548.

4 Benson MT, Nelson ME. Cyclocryotherapy: a review of cases over 10 year period. Br J Ophthalmol. 1990; 74: 103-105.

5 Coleman DJ, Lizzi FL, Driller J, Rosado AL, Chang S, Iwamoto T et al. Therapeutic ultrasound in the treatment of glaucoma. I. Experimental model. Ophthalmol 1985; 92: 339-346.

6 Coleman DJ, Lizzi FL, Driller J, Rosado AL, Burgess SE, Torpey $\mathrm{JH}$ et al. Therapeutic ultrasound in the treatment of glaucoma. II. Clinical applications. Ophthalmology 1985; 92: 347-353.

7 Beckman H, Kinoshita A, Rota AN, Sugar HS. Transscleral ruby laser irradiation of the ciliary body in the treatment of intractable glaucoma. Trans Am Acad Ophthalmol Otolaryngol 1972; 76: 423-436.

8 Assia EI, Hennis HL, Stewart WC, Legler UF, Carlson AN, Apple DJ. A comparison of neodymium:yttrium aluminium garnet and diode laser trans-scleral cyclophotocoagulation and cyclocryotherapy. Invest Ophthalmol Vis Sci 1991; 32: 2774-2778.

9 Brancato R, Leoni G, Trabucchi G, Cappellini A. Histopathology of continuous wave neodymium:yttrium aluminium garnet and diode laser contact laser trans-scleral lesions in rabbit ciliary body. A comparative study. Invest Ophthalmol Vis Sci 1991; 32: 1586-1592.

10 Cantor LB, Nicholas DA, Katz J, Moster MR, Poryzees E, Shields JA et al. Neodymium: YAG transcleral 
cyclophotocoagulation. The role of pigmentation. Invest Ophthalmol Vis Sci 1989; 30: 1834-1837.

11 Fankhauser F, van der Zypen E, Kwasniewska S, Rol P, England C. Transcleral cyclophotocoagulation using a neodymium:YAG laser. Ophthalmic Surg 1986; 17: 94-100.

12 Youn J, Cox TA, Herndon LW, Allingham RR, Shields MB. A clinical comparison of transscleral cyclophotocoagulation with neodymium:YAG and semiconductor diode lasers. Am J Ophthalmol 1998; 126: 640-647.

13 Hennis HL, Stewart WC. Semiconductor diode laser trans scleral cyclophotocoagulation in patients with glaucoma. Am J Ophthalmol 1992; 113: 81-85.

14 Hawkins TA, Stewart WC. One year results of semiconductor transcleral cyclophotocoagulation in patients with glaucoma. Arch Ophthalmol 1993; 111: 488-491.

15 Schlote T, Derse M, Rassman K, Nicaeus T, Dietz K, Thiel HJ. Efficacy and safety of transcleral diode laser cyclophotcoagulation for advanced glaucoma. J Glaucoma 2001; 10: 294-301.

16 Bloom PA, Tsai JC, Sharma K, Miller MH, Rice NS, Hitchings RA et al. 'Cyclodiode' trans-scleral diode laser cyclophotocoagulation in the treatment of advanced refractory glaucoma. Ophthalmology 1997; 104: 1508-1520.

17 Mistlberger A, Liebmann JM, Tschiderer H, Ritch R, Ruckhofer J, Grabner G. Diode laser transcleral cyclophotocoagulation for refractory gaucoma. J Glaucoma 2001; 10: 288-293.

18 Kosoko O, Gaasterland DE, Pollack IP, Enger CL. Long-term outcome of initial ciliary ablation with contact diode laser transscleral cyclophotocoagulation for severe glaucoma. The Diode Laser Ciliary Ablation Study Group. Ophthalmology 1996; 103: 1294-1302.

19 Murphy CC, Burnett CAM, Spry PGD, Broadway DC, Diamond JP. A two centre study of the dose response relation for transscleral diode laser cyclophotocoagula- tion in refractory glaucoma. Br J Ophthalmol 2003; 87: 1252-1257.

20 Gupta V, Aggarwal HC. Contact transscleral diode laser cyclophotocoagulation treatment for refractory glaucomas in Indian population. Indian J Ophthalmol 2000; 48: 295-300.

21 Chang SH, Chen YC, Li CY, Wu SC. Contact diode laser transclearal cyclophotocoagulation for refractory glaucoma: comparison of two treatment protocols. Can J Ophthalmol 2004; 39: 511-516.

22 Noureddin BN, ZEin W, Haddad C, Ma'luf R, Bashshur Z. Diode laser cyclophotocoagulation for refractory glaucoma: a 1 year follow-up of patients using an aggressive protocol. Eye 2006; 20: 329-335.

23 Walland MJ. Diode laser cyclophotocoagulation: longer term follow up of a standardized treatment protocol. Clin Exp Ophthalmol 2000; 28: 263-267.

24 Spencer AF, Vernon SA. 'Cyclodiode': results of a standard protocol. Br J Ophthalmol 1999; 83: 311-316.

25 Pastor SA, Singh K, Lee D, Juzycb MS, Lin SC, Netland PA et al. Cyclophotocoagulation. A report by the American Academy of Ophthalmology. Ophthalmology 2001; 108: 2130-2138.

26 Rebolleda G, Munoz FJ, Murube J. Audible pops during cyclodiode procedures. J Glaucoma 1999; 8: 177-183.

27 Schubert HD. The influence of exposure duration in transcleral ND:YAG laser cyclophotocoagulation. Am J Ophthalmol 1993; 115: 684-685.

28 Latina MA, Patel S, de Kater AW, Goode S, Nishioka NS, Puliafito CA. Transscleral cyclophotocoagulation using a contact laser probe: a histologic and clinical study in rabbits. Laser Surg Med 1989; 9: 437-438.

29 Kirwan JF, Shah P, Khaw PT. Diode laser cyclophotocoagulation: role in management of refractory pediatric glaucomas. Ophthalmology 2002; 109: 316-323.

30 Ansari E, Gandhewar J. Long-term efficacy and visual acuity following transcleral diode laser photocoagulation in cases of refatory and non-refractory glaucoma. Eye 2006; April 21 [E-pub ahead of print]. 